\title{
Toluene diisocyanate exposure induces airway inflammation of bronchial epithelial cells via the activation of transient receptor potential melastatin 8
}

\author{
Joo-Hee Kim ${ }^{1}$, Young-Sook Jang ${ }^{1}$, Seung-Hun Jang ${ }^{1}$, Ki-Suck Jung ${ }^{1}$, Seung-Hyun Kim², Young-Min Ye ${ }^{2}$ and \\ Hae-Sim Park ${ }^{2}$
}

Toluene diisocyanate (TDI) is the most important cause of occupational asthma (OA), and various pathogenic mechanisms have been suggested. Of these mechanisms, neurogenic inflammation is an important inducer of airway inflammation. Transient receptor potential melastatin 8 (TRPM8) is a well-established cold-sensing cation channel that is expressed in both neuronal cells and bronchial epithelial cells. A recent genome-wide association study of TDI-exposed workers found a significant association between the phenotype of TDI-induced OA and the single-nucleotide polymorphism rs10803666, which has been mapped to the TRPM8 gene. We hypothesized that TRPM8 located in airway epithelial cells may be involved in the pathogenic mechanisms of TDI-induced OA and investigated its role. Bronchial epithelial cells were treated with TDI in a dose- and timedependent manner. The expression levels of TRPM8 mRNA and protein were determined by quantitative real-time polymerase chain reaction and western blotting. TDI-induced morphological changes in the cells were evaluated by immunocytochemistry. Alterations in the transcripts of inflammatory cytokines were examined in accordance with TRPM8 activation by TDI. TRPM8 expression at both the mRNA and protein levels was enhanced by TDI in airway epithelial cells. TRPM8 activation by TDI led to significant increases in the mRNA of interleukin (IL)-4, IL-13, IL-25 and IL-33. The increased expression of the cytokine genes by TDI was partly attenuated after treatment with a TRPM8 antagonist. TDI exposure induces increased expression of TRPM8 mRNA in airway epithelial cells coupled with enhanced expression of inflammatory cytokines, suggesting a novel role of TRPM8 in the pathogenesis of TDI-induced OA.

Experimental \& Molecular Medicine (2017) 49, e299; doi:10.1038/emm.2016.161; published online 3 March 2017

\section{INTRODUCTION}

Toluene diisocyanate (TDI)-induced occupational asthma $(\mathrm{OA})$ is the most common type of OA worldwide. However, its pathogenesis is more complicated than other types of OA. ${ }^{1-3}$ Several studies have addressed the inflammatory process of isocyanate-induced $\mathrm{OA}$, describing heterogenic immunological pathways, such as Th1-controlled inflammation and Th2triggered allergic processes, as well as non-immunological pathways, such as epithelial injury, airway remodeling, oxidative stress generation and neurogenic inflammation. ${ }^{4}$ However, these mechanisms remain incompletely understood, and an ideal diagnostic marker has not been identified. Because TDI-induced OA has a poor prognosis with persistent asthmatic symptoms and a progressive decline in lung function, ${ }^{5}$ efforts have been made to identify individual risk factors for the development of this condition. ${ }^{1}$

The risk of TDI-induced OA is associated with a polymorphism of the neurokinin 2 receptor, a receptor for the neuropeptides that are released from activated airway sensory neurons, suggesting that neurogenic inflammation is involved in the development of TDI-induced $\mathrm{OA}^{6}{ }^{6}$ In addition, a genome-wide association study of TDI-exposed workers revealed that alpha T-catenin single-nucleotide polymorphisms (SNPs) were significantly associated with the phenotype of TDI-induced OA. Moreover, two SNPs of TRPM8 (rs10803666 and rs12434022, which have been mapped to the TRPM8 gene and are located on $2 \mathrm{q} 37$ and $14 \mathrm{q} 31$ ) showed significant associations using a recessive analysis model. ${ }^{7}$

\footnotetext{
${ }^{1}$ Division of Pulmonary, Allergy, and Critical Care Medicine, Department of Medicine, Hallym University Sacred Heart Hospital, Hallym University College of Medicine, Anyang, South Korea and ${ }^{2}$ Department of Allergy and Clinical Immunology, Ajou Research Institute for Innovation Medicine, Ajou University Medical Center, Suwon, South Korea

Correspondence: Professor H-S Park Department of Allergy and Clinical Immunology, Ajou Research Institute for Innovation Medicine, Ajou University Medical Center, 164, Worldcup-ro, Yeongtong-gu, Suwon 443-380, Korea.
}

E-mail: hspark@ajou.ac.kr

Received 22 August 2016; revised 3 October 2016; accepted 1 November 2016 
The TRPM8 is a nonselective calcium-permeable cation channel that is activated by cold temperatures of $<25^{\circ} \mathrm{C}$ and several chemicals, including the cooling agents menthol and icilin. ${ }^{8,9}$ TRPM8 is expressed on a subset of sensory neurons from the dorsal root ganglion and trigeminal ganglia, as well as in a number of non-neuronal areas. Recent studies have shown that activation of the TRPM8 variant in lung epithelial cells by cold air leads to increased expression of proinflammatory cytokine genes, such as interleukin (IL)-6 and IL-8. ${ }^{10}$ Moreover, TRPM8-mediated mucus hypersecretion has been demonstrated in patients with chronic obstructive pulmonary disease, ${ }^{11}$ suggesting that TRPM8 is potentially involved in chronic airway inflammation.

Epithelial cells form a continuous lining along the airways, providing a protective barrier between the external and internal environments. In addition to forming a barrier, the epithelium has the ability to generate a wide range of mediators that can modulate inflammatory responses, helping to either maintain homeostasis or enhance inflammation. ${ }^{12}$ The asthmatic airway epithelium is a major source of other cytokines and chemokines, and increased susceptibility to injury and altered repair are important for both airway remodeling and the mucous metaplastic responses in patients with chronic asthma. ${ }^{13,14}$

The goal of the present study was to explore the hypothesis that TDI exposure can cause changes in airway epithelial cells by activating TRPM8 and that this TDI-induced TRPM8 activation induces airway inflammation.

\section{MATERIALS AND METHODS}

\section{Chemicals}

Toluene-2, 4-diisocyanate, N-(4-tert-butylphenyl)-4-(3-chloropyridin2-yl) piperazine-1-carboxamide (BCTC), menthol and dexamethasone were purchased from Sigma Chemical. (St. Louis, MO, USA). Rabbit anti-TRPM8 (C-term) polyclonal antibody was purchased from Abcam (Cambridge, UK). Oligonucleotides were purchased from Bioneer (Daejeon, Republic of Korea). SYBR Premix EX Taq was purchased from TaKaRa Biotechnology (Shiga, Japan).

\section{Cell culture}

Human bronchial epithelial cells (cell line BEAS-2B) were used in this study (CRL-9609; American Type Culture Collection, Rockville, MD, USA). BEAS-2B cells were cultured in RPMI-1640 medium (Invitrogen, Carlsbad, CA, USA) with $10 \%$ fetal bovine serum and maintained at $37^{\circ} \mathrm{C}$ in a humidified atmosphere of $5 \%$ carbon dioxide and $95 \%$ air. The cells were maintained at $37^{\circ} \mathrm{C}$, between 30 and $90 \%$ confluence, in an air-ventilated and humidified incubator maintained with 5\% carbon dioxide. For subculturing, the cells were trypsindissociated and passaged every 2-4 days.

\section{Conventional real-time polymerase chain reaction of TRPM8}

Sense and antisense polymerase chain reaction (PCR) primers specific to TRPM8 were used: sense, 5'-CAGACCCCTGGGTACATGGTGGA TG-3'; antisense, 5'-GCCTTTCAAGGTTGCATTTTG GGCGAC-3'. PCR reactions were carried out using AccuPower PCR Premix (Bioneer, Daejeon, Republic of Korea) with the following parameters: denaturation at $94^{\circ} \mathrm{C}$ for $30 \mathrm{~s}$, annealing at $62^{\circ} \mathrm{C}$ for $30 \mathrm{~s}$ and extension at $72{ }^{\circ} \mathrm{C}$ for $30 \mathrm{~s}$. Thirty-five cycles were performed, followed by a final extension at $72{ }^{\circ} \mathrm{C}$ for $7 \mathrm{~min}$; the products were then stored at $4{ }^{\circ} \mathrm{C}$. PCR products were analyzed by electrophoresis with $1.8 \%$ agarose gel and visualized by ethidium bromide staining. A 180-nt portion of $\beta$-actin cDNA was simultaneously amplified by PCR using the following primers: sense, 5'-GACAACGGCTCCGGCATGTGCA -3'; antisense, 5'-TGAGGATGCCTCTCTTGCTCTG-3'. $\beta$-actin was used as an internal standard to normalize the PCR product intensities between samples.

\section{Quantitative real-time PCR}

BEAS-2B cells were subcultured into $25-\mathrm{cm}^{2}$ flasks and grown to approximately $95 \%$ confluence. Cells were exposed to either TDI or menthol for $3 \mathrm{~h}$. The TRPM8 antagonist BCTC or dexamethasone was added to cells $5 \mathrm{~min}$ before treatment. cDNA from the treated cells was obtained as described above. cDNA corresponding to human interleukin IL-4, $-6,-8,-13,-25$ and -33 was amplified by PCR from $1 \mu \mathrm{l}$ of the cDNA synthesis reaction using SYBR Premix Ex Taq (TaKaRa Biotechnology) and the following primers:

IL-4: sense, 5'-CCAACTGCTTCCCCCTCTG-3'; antisense, 5'-TCT GTTACGGTCAACTCGGTG-3'.

IL-6: sense, 5'-TGTTCGTCATGGGTGTGAACC-3'; antisense, 5'-AACTCCAAAAGACCAGTGATGATTT-3'.

IL-8: sense, 5'-CTGGCCGTGGCTCTCTTG-3'; antisense, 5'-TTA GCACTCCTTGGCAAAACTG-3'.

IL-13: sense, 5'-CCTCATGGCGCTTTTGTTGAC-3'; antisense, 5'-TCTGGTTCTGGGTGATGTTGA-3'.

IL-25: sense, 5'-CCAGGTGGTTGCATTCTTGG-3'; antisense, 5'-TGGCTGTAGGTGTGGGTTCC-3'.

IL-33: sense, 5'-CAAAGAAGTTTGCCCCATGT-3'; antisense, 5'-AAGGCAAAGCACTCCACAGT-3'.

Glyceraldehyde-3-phosphate dehydrogenase (GAPDH) was simultaneously amplified as an internal standard. Experiments were reproduced a minimum of three times with different passages of cells.

\section{Immunocytochemistry}

BEAS-2B cells were cultured on a 22-mm round cover slip (BD Biosciences, Bedford, MA, USA) until 75\% confluence and fixed with $4 \%$ paraformaldehyde in phosphate-buffered saline (PBS) for $10 \mathrm{~min}$. The cells were then washed three times with PBS, and nonspecific binding was blocked using a solution of $5 \%$ bovine serum albumin in PBS. The cells were rinsed three times with PBS and incubated at room temperature for $2 \mathrm{~h}$ with a rabbit polyclonal IgG antibody fraction specific to human TRPM8 (Abcam), which was diluted 1:250 in the blocking solution. The cells were washed and treated for $1 \mathrm{~h}$ at room temperature with an Alexa-Fluor 488 conjugated goat antirabbit IgG secondary antibody (Molecular Probes, Eugene, OR, USA) at a dilution of 1:500 in the blocking solution. The nuclei were counterstained blue using 4', 6-diamidino-2-phenylindole (DAPI) at $300 \mathrm{~nm}$ dilution in PBS. Controls consisted of untreated cells or cells treated with either primary or secondary antibodies alone. Images were collected using a ZEISS LSM700 laser scanning confocal microscope (Carl Zeiss, Oberkochen, Germany) equipped with filters to visualize green fluorescent protein and DAPI. The immunoreactivity of TRPM8 was detected as green fluorescence.

\section{Statistical analysis}

Statistical analysis was performed using ANOVA and Dunnett's multiple-comparison post-test, with a significance level of $P<0.05$. The paired $t$-test with a significance level of $P<0.05$ was also used. 
a

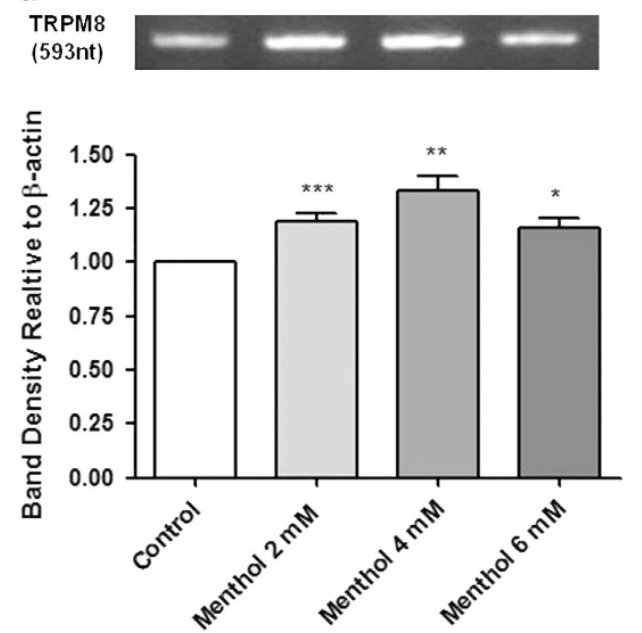

b
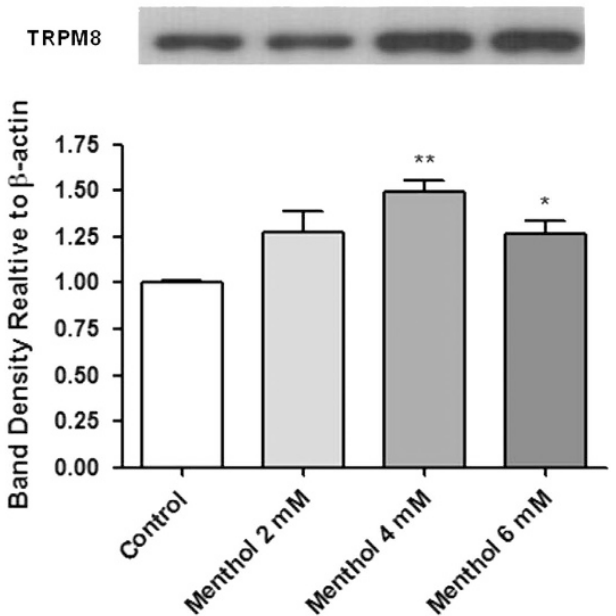

Figure 1 Expression of TRPM8 (a) mRNA and (b) protein in lung epithelial cells. Agarose gel demonstrating the presence of a 583-nt PCR product from a human bronchial epithelial cell line (BEAS-2B). The normalized ( $\beta$-action) PCR product intensities are shown below. Menthol (2-6 mm) treatment for $3 \mathrm{~h}$ induced increases in both TRPM8 mRNA and protein expression. Each bar corresponds to the mean normalized product intensity and s.d. An asterisk represents statistically significant increases in mRNA and protein expression relative to untreated cells (paired $t$-test, ${ }^{*} P<0.05,{ }^{* *} P<0.01,{ }^{* * *} P<0.001$ ).
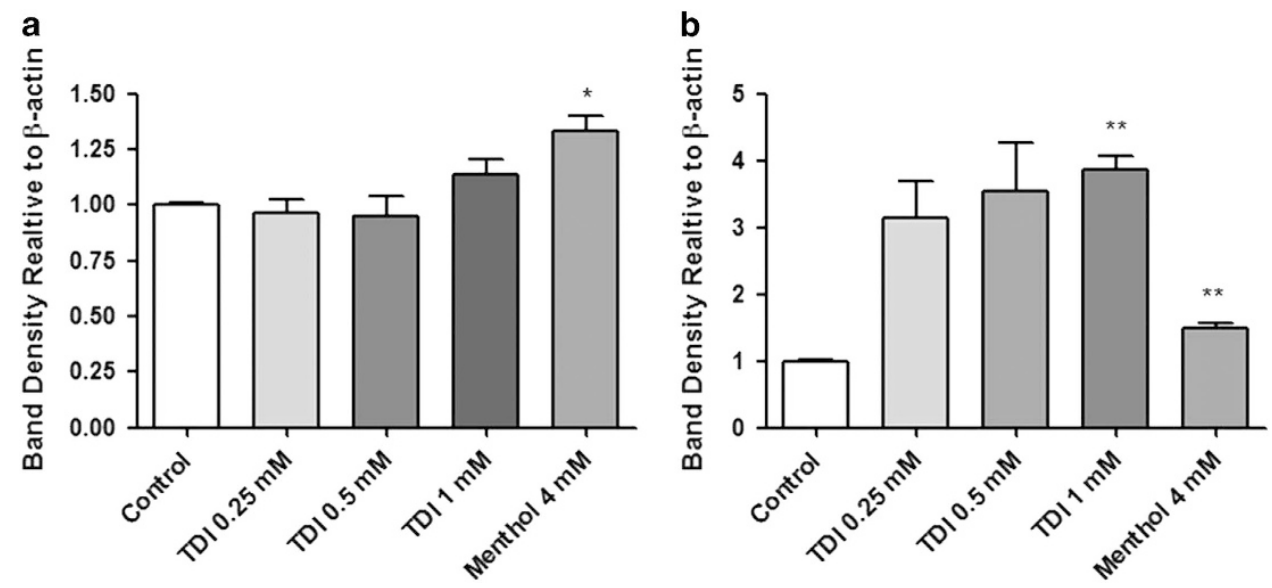

Figure 2 Expression of TRPM8 (a) mRNA and (b) protein after treatment with toluene diisocyanate $(0.25-1.0 \mathrm{~mm})$ and menthol (4.0 mm) for $3 \mathrm{~h}$ in BEAS-2B cells. Each bar corresponds to the mean normalized product intensity and s.e. An asterisk represents statistically significant increases in mRNA and protein expression relative to untreated cells (paired $t$-test, ${ }^{*} P<0.05,{ }^{*} P<0.01$ ).

\section{RESULTS}

\section{Identification of TRPM8 transcript and protein in lung} epithelial cells

We investigated whether TRPM8 is expressed on BEAS-2B cells. Expression of TRPM8 was identified using RT-PCR. Figure 1 shows the amplified PCR products generated after 35 cycles and the TRPM8 protein expression using western blot analysis. TRPM8 activation by menthol for $3 \mathrm{~h}$ led to significant increases in both the TRPM8 transcript and protein in a dose-dependent manner.

\section{Effect of TDI on TRPM8 transcripts and protein in lung epithelial cells}

To determine whether TDI can activate TRPM8, the TRPM8 transcript and protein were measured after treatment with TDI $(0.25-1 \mathrm{mM})$, and the TRPM8 agonist menthol $(2-6 \mathrm{mM})$ was used as a positive control. TDI tended to increase the TRPM8 transcript, but this change did not reach statistical significance. However, TDI led to a robust increase in the protein level, which was significantly higher than that achieved with menthol treatment $(P<0.01)$ (Figure 2).

\section{Expression of TRPM8 protein in lung epithelial cells}

The expression of TRPM8 protein in BEAS-2B cells was evaluated by immunocytochemical analysis using a polyclonal antibody for human TRPM8 (Figure 3). Without any treatment, TRPM8 protein was not detected. However, after treatment with TDI or menthol, time- and dose-dependent intense green staining was detected. The staining pattern suggested localization of the TRPM8 to the plasma membrane (peripheral staining) and endoplasmic reticulum (perinuclear staining). 
a

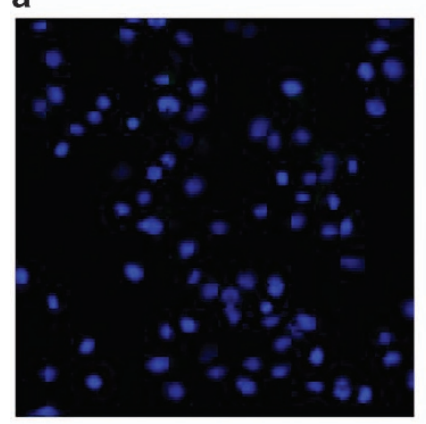

$0 \mathrm{~min}$

b

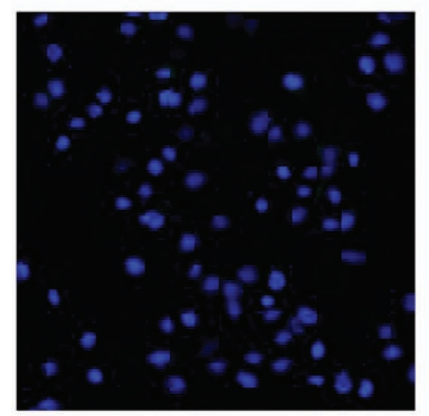

$3 \mathrm{hr}$ control

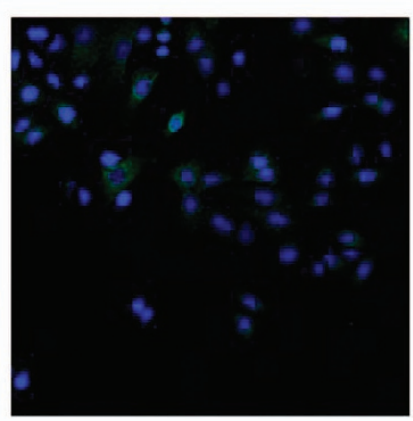

$10 \mathrm{~min}$

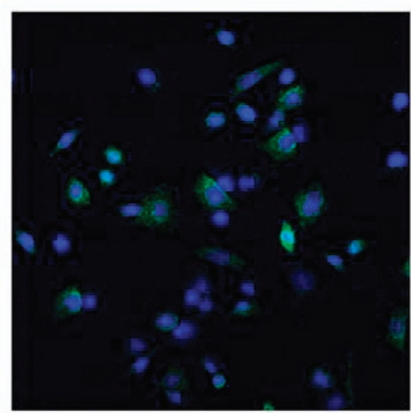

TDI $0.25 \mathrm{mM}$

C

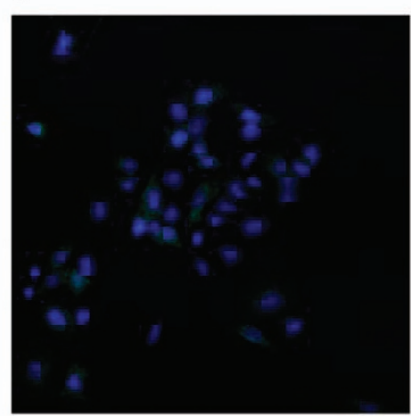

Menthol 2mM

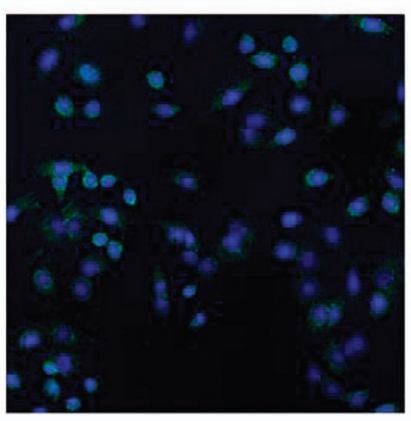

$60 \mathrm{~min}$

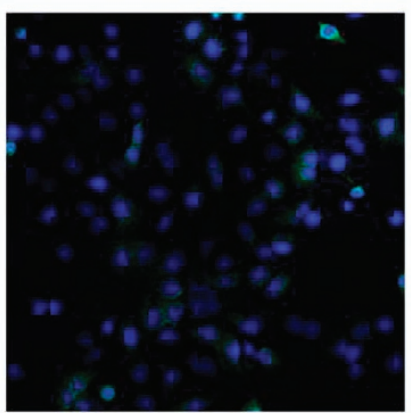

TDI $0.5 \mathrm{mM}$

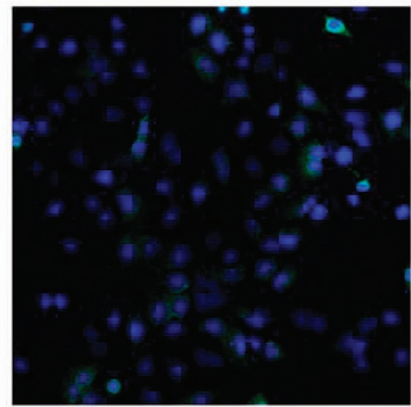

Menthol 4mM

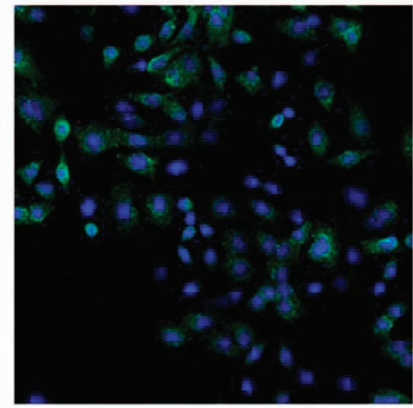

$180 \mathrm{~min}$

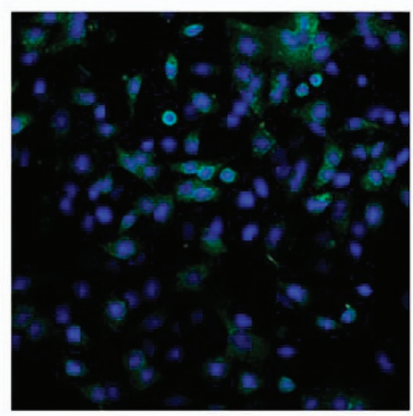

TDI 1mM

Figure 3 Immunocytochemical detection of TRPM8 protein in BEAS-2B cells. Immunocytochemical staining using a polyclonal antibody of TRPM8 demonstrated protein expression in BEAS-2B cells. The nuclei were counterstained blue using DAPI. (a) 1 mM of TDI intensified green staining in the plasma membranes in a time-dependent manner. (b) Pretreatment with TDI (0.25-1 mm) or (c) menthol (2-4 mm) for $3 \mathrm{~h}$ led to increased green staining in a dose-dependent manner. DAPI, 4', 6-diamidino-2-phenylindole; TDI, toluene diisocyanate.

Inhibition of TRPM8 transcript and protein by BCTC in lung epithelial cells

To test the hypothesis that TDI selectively activates TRPM8 on bronchial epithelial cells, BEAS-2B cells were treated with TDI after pretreatment with BCTC, an antagonist of TRPM8, at $50-100 \mu \mathrm{M}$. Increased TRPM transcripts by TDI were attenuated by BCTC (Figure 4a). Likewise, 50-100 $\mu \mathrm{m} \mathrm{BCTC}$ blocked the TDI-induced increase in TRPM8 protein expression (Figure 4b).

Effect of TRPM8 activation on cytokine gene expression in lung epithelial cells

The coupling of TRPM8 activation in BEAS-2B cells with enhanced expression of proinflammatory cytokines (IL-6 and
IL-8), epithelial-driven cytokines (IL-25 and IL-33) and Th2 cytokines (IL-4 and IL-13) was evaluated following treatment with TDI. No alteration was observed in IL-6 and IL-8 transcripts after TDI exposure. Instead, co-treatment with TDI and BCTC resulted in significantly lower IL-6 and IL-8 transcripts compared with the control (Figure 5). The degree of decrement in IL- 6 by BCTC was similar to that by dexamethasone. The TRPM8 activation by TDI led to a significant increase in the transcripts for IL-4, IL-13 and IL-25 (Figures 6 and 7). The transcript for IL-33 tended to increase with $1 \mathrm{~mm}$ of TDI, but this change did not reach statistical significance. TDI-induced increases in cytokines gene expression were partly attenuated after co-treatment with BCTC and dexamethasone (Figures 6 and 7). 

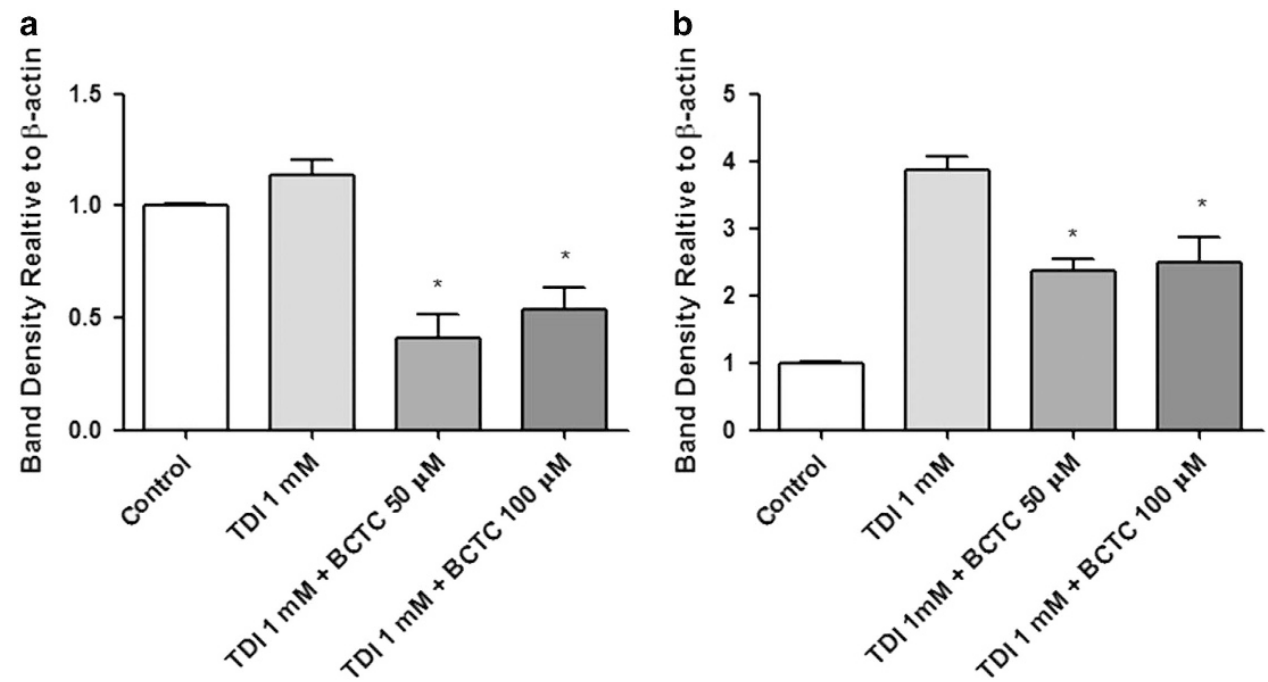

Figure 4 Inhibition of TDI-induced activation of the TRPM8 in BEAS-2B cells at the (a) mRNA and (b) protein levels. Each bar represents the mean values normalized to untreated controls for the cell populations and standard deviations. Values significantly different from cells treated with TDI $(1 \mathrm{~mm})$ are indicated by an asterisk (paired $t$-test, $\left.{ }^{*} P<0.05\right)$. TDI, toluene diisocyanate.
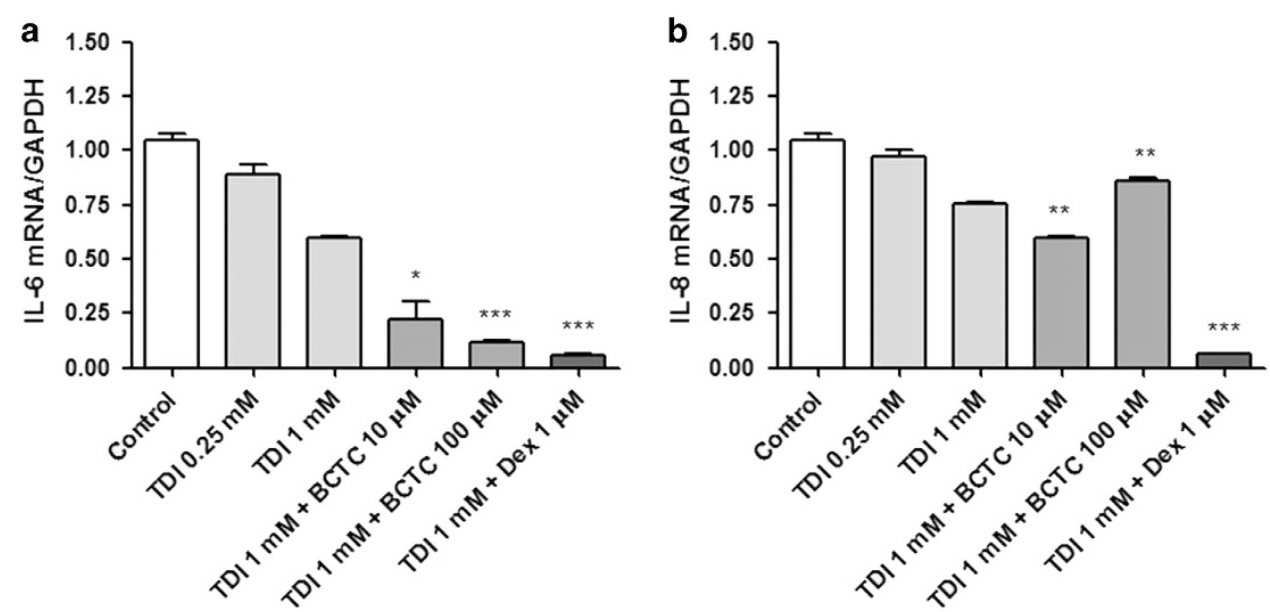

Figure 5 TRPM8-mediated alterations in (a) IL-6 and (b) IL-8 transcription in BEAS-2B cells. Each bar represents the mean values normalized to untreated controls for the cell populations and s.d. Values significantly different from cells treated with TDI (1 mm) are indicated by an asterisk (paired $t$-test, ${ }^{*} P<0.05,{ }^{*} P<0.01,{ }^{* *} P<0.001$ ).

\section{DISCUSSION}

In the current study, we demonstrated that TRPM8 is activated by TDI and that activation of this receptor is involved in airway inflammation involving lung epithelial cells.

TDI, which is a reactive compound widely used in the manufacturing of polyurethane foams and coatings, can cause respiratory symptoms, such as coughing, rhinitis, dyspnea and chest tightness, in exposed workers. ${ }^{4}$ It can act as an irritant as well as a strong sensitizer; as a sensitizer, driven by TDI-specific antibodies, it promotes airway inflammation via the immunological pathway. As an irritant material, TDI leads to stimulation of trigeminal nerve endings in airways, thereby inducing the local release of tachykinin neuropeptides, such as substance P, neurokinin A and calcitonin-gene-related peptide. These findings suggest that TRP receptors may be involved in neurogenic inflammation of the airways in patients with TDI-induced OA. ${ }^{15}$ Some studies have investigated the role of TRP receptors in TDI-OA. Taylor-Clark et al. ${ }^{16}$ showed that TRPA1 is a molecular transducer through which TDI causes sensory nerve activation and respiratory reflexes in an animal model. Devos et al. ${ }^{17}$ recently demonstrated that TDI can directly activate TRPA1 and that both TRPA1 and mast cells in airways are critical for the induction of airway hyperresponsiveness in an animal model of TDI-induced OA.

A previous genome-wide association study of TDI-exposed workers showed that two SNPs (rs10803666 and rs12434022) of TRPM8 had significant associations with the phenotype of TDI-induced OA with higher odds ratios, indicating strong correlations. ${ }^{7}$ TRPM8 is expressed on a subpopulation of airway vagal afferent nerves, pulmonary arterial smooth muscle cells and lung epithelial cells. Sabnis et al. ${ }^{10}$ showed that a TRPM8 variant manipulated for stable overexpression of TRPM8 was expressed primarily within endoplasmic reticulum membranes of lung epithelial cells. In the present study, 

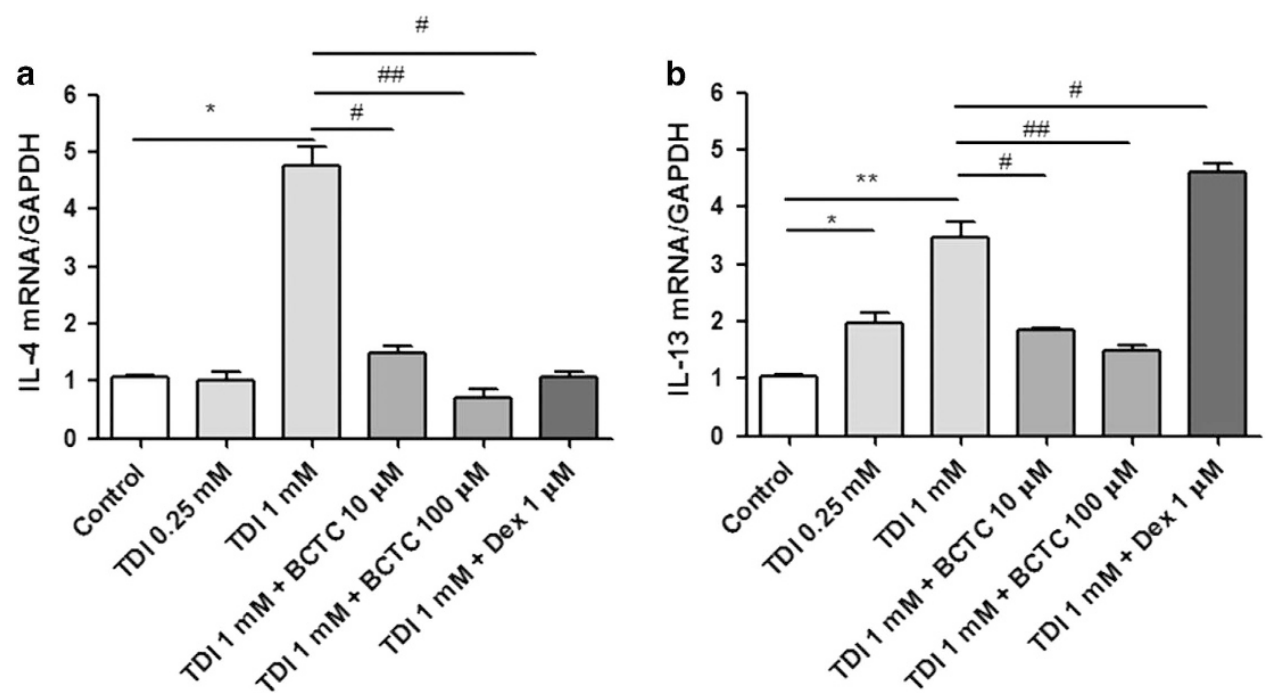

Figure 6 TRPM8-mediated alteration in (a) IL-4 and (b) IL-13 transcription in BEAS-2B cells. Each bar represents the mean values normalized to untreated controls for the cell populations and standard deviations. Values significantly different from untreated and TDI ( $1 \mathrm{~mm}$ )-treated cells are indicated by * and ${ }^{*}$, respectively (paired $t$-test, *, ${ }^{*}, P<0.05 ;{ }^{*}, \# \#, P<0.01$ ). TDI, toluene diisocyanate.
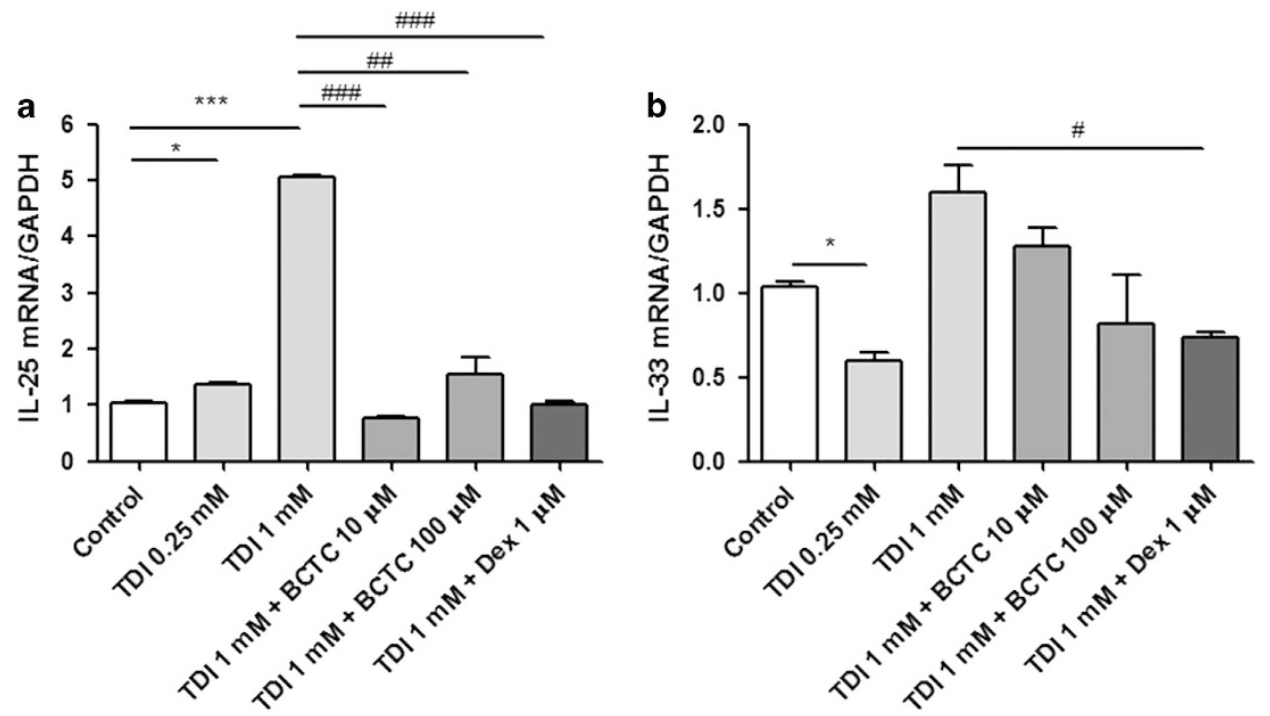

Figure 7 TRPM8-mediated alteration in (a) IL-25 and (b) IL-33 transcription in BEAS-2B cells. Each bar represents the mean values normalized to untreated controls for the cell populations and standard deviations. Values significantly different from untreated and TDI (1 mm)-treated cells are indicated by * and ${ }^{\#}$, respectively (paired $t$-test, * ${ }^{*}, P<0.05 ; * *, \# \#, P<0.01 ;{ }^{* * *}, \# \#, P<0.001$ ). TDI, toluene diisocyanate.

we demonstrated that TRPM8 was spontaneously expressed in human bronchial epithelial cell lines without the use of a TRPM8 variant and that TDI itself can cause TRPM8 activation comparative to its agonist, menthol. These findings suggest that TDI exposure can induce TRPM8 activation in the airway epithelial cells of TDI-exposed workers.

To determine whether TRPM8 activation by TDI is associated with airway inflammation in TDI-induced OA, we examined the expression of several cytokine genes, including proinflammatory cytokines, such as IL-6 and IL-8; Th2 cytokines, including IL-4 and IL-13; and epithelial-driven cytokines, such as IL-25 and IL-33. Mattoli et al. ${ }^{18}$ demonstrated that IL-6 was released from TDI-exposed airway epithelial cells and that it consecutively induced T-cell activation and proliferation. By releasing IL-8, a potent activating and chemotactic factor of neutrophils, diisocyanate-exposed epithelial cells enhance the infiltration of neutrophils and affect smooth muscle cells. ${ }^{19,20}$ This indicates that IL-6 and IL-8 are directly involved in airway inflammation and bronchial remodeling in patients with TDI-induced OA. When we co-treated BEAS-2B cells with TDI and a TRPM8 antagonist, we found significant reductions in both the IL-6 and IL-8 transcripts, and the degree of decrement was similar to that with dexamethasone. These results suggest that ongoing inflammation of airway epithelial cells by TDI exposure is suppressed by TRPM8 antagonists and that TRPM8 can be a 
key contributor to airway inflammation in patients with TDI-induced OA.

Several studies have shown infiltration of inflammatory cells in the airway mucosa, especially eosinophils, $\mathrm{CD}^{+}{ }^{+}$cells and mast cells, in both animal models and human airways affected by TDI-induced OA. T cells and eosinophils have been shown to release various cytokines, including IL-4 and IL-5, to induce immune responses. ${ }^{4}$ In the present study, we found that TRPM8 activation by TDI was coupled with enhanced expression of IL-4 and IL-13 mRNA from bronchial epithelial cells. Moreover, treatment with a TRPM8 antagonist attenuated the expression of IL-4 and IL-13 mRNA levels, suggesting that bronchial epithelial cells have a role in the induction of Th2-type inflammation in TDI-induced OA. However, the mechanism by which TRPM8 activation by TDI induces Th2 inflammation in bronchial epithelial cells remains unknown. Considering that TDI is redox-active and capable of inducing oxidative stress that includes inflammation and cell death, ${ }^{21,22}$ and considering that production of reactive oxygen species by TPRM8 agonists is dependent on TRPM8-mediated calcium entry, ${ }^{23}$ we postulate that TDI induces the production of reactive oxygen species in airway epithelial cells followed by TRPM8 activation and Th2-cytokine release from airway epithelial cells.

Airway epithelial cells constitutively express IL-25. On exposure to allergens with proteolytic activities or viruses, the release of IL-25 mRNA and IL-25 protein from bronchial epithelial cells increases. ${ }^{24}$ IL-25 reportedly induces type 2 innate lymphoid cells and $\mathrm{CD}^{+}{ }^{+} \mathrm{T}$ cells to produce IL-4, IL-5 and IL-13 and has a critical role in airway eosinophilia, mucus overproduction and airway remodeling. ${ }^{25}$ In the present study, we found that IL-25 mRNA increased along with TRPM8 activation by TDI and observed a positive correlation between IL-25 mRNA and IL-4 or IL-13 mRNA levels. IL-33 is an inducer of Th2 adaptive immunity. It is released by damaged epithelial cells during injury or necrosis caused by various triggers, such as viruses, allergens and smoke. ${ }^{26}$ Although the change was not statistically significant, IL-33 mRNA tended to be increased in accordance with TRPM8 activation by TDI and attenuated with the TRPM8 antagonist in this study. This is the first study to show an association between increases in epithelial-driven cytokines and TRPM8 activation by TDI in an in vitro model, which is a novel function of TRPM8 in the pathogenic mechanism of TDI-induced OA. However, further studies are needed to reveal the pathway for release of IL-25 or -33 by airway epithelial cells.

In conclusion, our results demonstrate that TDI exposure can induce TRPM8 activation in bronchial epithelial cells. TRPM8 activation by TDI enhanced airway inflammation similar to that induced by Th2 cytokines (IL-4 and IL-13) and epithelial-driven cytokines (IL-25 and IL-33), and this was partly attenuated with a TRPM8 antagonist. The role of TRPM8 in inflammatory responses involving bronchial epithelial cells will serve as a foundation for further development of new therapeutic targets for patients with TDI-induced OA.

\section{CONFLICT OF INTEREST}

The authors declare no conflict of interest.

\section{ACKNOWLEDGEMENTS}

This study was supported by a grant of the Korea Health Technology R\&D Project through the Korea Health Industry Development Institute, funded by the Ministry of Health \& Welfare, Republic of Korea (HI14C2628). This research was supported by a grant from Hallym University Medical Center Research Fund (01-2012-12) and a grant from the Korean Society of Occupational Asthma and Lung Diseases.

1 Palikhe NS, Kim JH, Park HS.. Biomarkers predicting isocyanateinduced asthma. Allergy Asthma Immunol Res 2011; 3: 21-26.

2 Kwon SC, Song J, Kim YK, Calvert GM.. Work-related asthma in korea findings from the Korea Work-Related Asthma Surveillance (KOWAS) program, 2004-2009. Allergy Asthma Immunol Res 2015; 7: 51-59.

3 Fajt ML, Wenzel SE.. Development of new therapies for severe asthma. Allergy Asthma Immunol Res 2016; 8: e16.

4 Shin YS, Kim MA, Pham LD, Park HS. Cells and mediators in diisocyanateinduced occupational asthma. Curr Opin Allergy Clin Immunol 2013; 13: 125-131.

5 Ruegger M, Droste D, Hofmann M, Jost M, Miedinger D.. Diisocyanateinduced asthma in Switzerland: long-term course and patients' selfassessment after a 12-year follow-up. J Occup Med Toxicol 2014; 9: 21.

6 Ye YM, Kang YM, Kim SH, Kim CW, Kim HR, Hong CS et al. Relationship between neurokinin 2 receptor gene polymorphisms and serum vascular endothelial growth factor levels in patients with toluene diisocyanateinduced asthma. Clin Exp Allergy 2006; 36: 1153-1160.

$7 \mathrm{Kim}$ SH, Cho BY, Park CS, Shin ES, Cho EY, Yang EM et al. Alpha-Tcatenin (CTNNA3) gene was identified as a risk variant for toluene diisocyanate-induced asthma by genome-wide association analysis. Clin Exp Allergy 2009; 39: 203-212.

8 Grace MS, Baxter M, Dubuis E, Birrell MA, Belvisi MG.. Transient receptor potential (TRP) channels in the airway: role in airway disease. $\mathrm{Br} J$ Pharmacol 2014; 171: 2593-2607.

9 Laing RJ, Dhaka A.. ThermoTRPs and pain. Neuroscientist 2016; 22: 171-187.

10 Sabnis AS, Shadid M, Yost GS, Reilly CA.. Human lung epithelial cells express a functional cold-sensing TRPM8 variant. Am J Respir Cell Mol Biol 2008; 39: 466-474.

11 Li M, Li Q, Yang G, Kolosov VP, Perelman JM, Zhou XD.. Cold temperature induces mucin hypersecretion from normal human bronchial epithelial cells in vitro through a transient receptor potential melastatin 8 (TRPM8)mediated mechanism. J Allergy Clin Immunol 2011; 128: 626-634.

12 Lambrecht BN, Hammad H.. The airway epithelium in asthma. Nat Med 2012; 18: 684-692.

13 Lloyd CM, Saglani S.. Epithelial cytokines and pulmonary allergic inflammation. Curr Opin Immunol 2015; 34: 52-58.

14 Kim B-G, Lee P-H, Lee S-H, Kim Y-E, Shin M-Y, Kang Y et al. Long-term effects of diesel exhaust particles on airway inflammation and remodeling in a mouse model. Allergy Asthma Immunol Res 2016; 8: 246-256.

15 Mapp CE, Lucchini RE, Miotto D, Chitano P, Jovine L, Saetta M et al. Immunization and challenge with toluene diisocyanate decrease tachykinin and calcitonin gene-related peptide immunoreactivity in guinea pig central airways. Am J Respir Crit Care Med 1998; 158: 263-269.

16 Taylor-Clark TE, Kiros F, Carr MJ, McAlexander MA.. Transient receptor potential ankyrin 1 mediates toluene diisocyanate-evoked respiratory irritation. Am J Respir Cell Mol Biol 2009; 40: 756-762.

17 Devos FC, Boonen B, Alpizar YA, Maes T, Hox V, Seys S et al. Neuroimmune interactions in chemical-induced airway hyperreactivity. Eur Respir J 2016; 48: 380-392.

18 Mattoli S, Miante S, Calabro F, Mezzetti M, Fasoli A, Allegra L.. Bronchial epithelial cells exposed to isocyanates potentiate activation and proliferation of T-cells. Am J Physiol 1990; 259: L320-L327.

19 Lee YM, Kim HA, Park HS, Lee SK, Nahm DH.. Exposure to toluene diisocyanate (TDI) induces IL-8 production from bronchial epithelial cells: effect of pro-inflammatory cytokines. J Korean Med Sci 2003; 18: 809-812. 
20 Kuo PL, Huang MS, Huang SK, Ni WC, Hung JY, Ko YC et al. Signalling pathway of isophorone diisocyanate-responsive interleukin-8 in airway smooth muscle cells. Eur Respir J 2011; 37: 1226-1236.

21 Lange RW, Day BW, Lemus R, Tyurin VA, Kagan VE, Karol MH. Intracellular S-glutathionyl adducts in murine lung and human bronchoepithelial cells after exposure to diisocyanatotoluene. Chem Res Toxicol 1999; 12: 931-966.

22 Hur GY, Kim SH, Park SM, Ye YM, Kim CW, Jang AS et al. Tissue transglutaminase can be involved in airway inflammation of toluene diisocyanate-induced occupational asthma. J Clin Immunol 2009; 29: 786-794.

23 Zhu S, Wang Y, Pan L, Yang S, Sun Y, Wang X et al. Involvement of transient receptor potential melastatin-8 (TRPM8) in menthol-induced calcium entry, reactive oxygen species production and cell death in rheumatoid arthritis rat synovial fibroblasts. Eur J Pharmacol 2014; 725: $1-9$.

24 Cheng D, Xue Z, Yi L, Shi H, Zhang K, Huo X et al. Epithelial interleukin-25 is a key mediator in Th2-high, corticosteroid-responsive asthma. Am J Respir Crit Care Med 2014; 190: 639-648.
25 Gregory LG, Jones CP, Walker SA, Sawant D, Gowers KH, Campbell GA et al. IL-25 drives remodelling in allergic airways disease induced by house dust mite. Thorax 2013; 68: 82-90.

26 Makrinioti H, Toussaint M, Jackson DJ, Walton RP, Johnston SL.. Role of interleukin 33 in respiratory allergy and asthma. Lancet Respir Med 2014; 2: $226-237$.

(1) () $\odot$ This work is licensed under a Creative Commons Attribution-NonCommercial-NoDerivs 4.0 International License. The images or other third party material in this article are included in the article's Creative Commons license, unless indicated otherwise in the credit line; if the material is not included under the Creative Commons license, users will need to obtain permission from the license holder to reproduce the material. To view a copy of this license, visit http://creativecommons.org/licenses/by-nc-nd/4.0/ 